\title{
Mesenchymal stem cell spheroids: potential cell resource for cell therapy
}

\author{
Zhongjuan $\mathrm{XU}^{1,2}$, Xingzhi LIU ${ }^{1,2}, \mathrm{Yu} \mathrm{WEI}^{3}$, Zhe ZHAO ${ }^{2}$, Junjun $\mathrm{CAO}^{1,2}$, Yong QIAO ${ }^{2}$, Yanzhen $\mathrm{YU}^{1,2}$, \\ Junjie $\mathrm{ZHONG}^{4}$, Guangli SUO ${ }^{2 *}$ \\ ${ }^{1}$ School of Nano-Tech and Nano-Bionics, University of Science and Technology of China, Hefei, Anhui 230026, China. \\ ${ }^{2}$ CAS Key Laboratory of Nano-Bio Interface, Suzhou Institute of Nano-Tech and Nano-Bionics, Chinese Academy of Sciences, Jiangsu 215123, China. \\ ${ }^{3}$ Livingchip Lnc., Nanjing, Jiangsu 211112, China. \\ ${ }^{4}$ Department of Neurosurgery, Fudan University Huashan Hospital, National Key Laboratory of Medical Neurobiology, Institutes of Brain Science, Shanghai \\ Medical College, Fudan University, Shanghai 200041, China.
}

*Correspondence: glsuo2013@sinano.ac.cn

https://doi.org/10.37175/stemedicine.v2i5.67

\begin{abstract}
Mesenchymal stromal/stem cells (MSCs) have been applied in an increasing number of clinical trials in recent years. MSCs have many advantages in cell therapy for regenerative medicine for their extensive sources, low immunogenicity, self-renewal ability and multilineage differentiation potential. However, the clinical application of MSCs is still confronted by many challenges including low survival ability in vivo, the loss of main original characteristics due to two-dimensional (2D) culture for fast expansion, and the lack of technologies for mass production of high-quality MSCs. Three-dimensional (3D) culture has been widely regarded as a more preferable and closer physiological microenvironment for cell survival, growth and behavior, because 3D culture can artificially create an environment allowing cells to interact with their surroundings in complete three dimensions. Recently, many different 3D spheroid culture methods have been developed to optimize the biological characteristics of MSCs to meet the demand of regenerative medicine. In this review, we comprehensively discuss the merits and demerits of different spheroid formation methods, expound the mechanisms of spheroid formation and its microenvironment, and illustrate their optimized biological functions and the pre-clinical applications in tissue injury and regeneration. In the end, we prospect the trends of this research field and propose the key problems needed to be solved in the future.
\end{abstract}

Keywords: Mesenchymal stromal/stem cells - Three-dimensional culture · Spheroids · Stem cell optimization Regenerative medicine

\section{Introduction}

Mesenchymal stromal/stem cells (MSCs) are nonhematopoietic multipotent stem cells originated from the mesoderm. Since MSCs were initially isolated from bone marrow by Friedenstein et al. in 1970 (1), nowadays, they have been easily separated from a variety of adult or embryonic tissues including bone marrow, adipose tissue, umbilical cord, amnion, placenta, umbilical cord blood and dental pulp, and can be expanded successfully in vitro (2-7).

Received: Sep 14, 2020; Accepted: Dec 8, 2020.

() The Author(s). 2020 This is an Open Access article distributed under the terms of the Creative Commons License (http://creativecommons.org/licenses/by/4.0/) which permits unrestricted use, distribution, and reproduction in any medium or format, provided the original work is properly cited.
MSCs are characterized by their abilities of self-renewal, multilineage differentiation, extensive proliferation and paracrine. Moreover, MSCs lack immunogenicity and have the homing ability to migrate to sites of tissue injury in response to inflammatory factors by intravenous administration (8). They modulate the immune response and inhibit the inflammation by secreting multiple bioactive factors to create a niche which promotes the recovery of injured cells and angiogenesis $(9,10)$. In order to easily compare and contrast the increasing data among different studies, the Mesenchymal and Tissue Stem Cell Committee of the International Society for Cellular Therapy proposed minimal criteria to define human MSCs (hMSCs) in 2006 (11). MSCs would keep adherence to plastic under standard culture conditions with the capacity 
to differentiate into osteoblasts, chondrocytes and adipocytes in vitro, and express specific surface markers CD73, CD90 and CD105, while lack the expression of several hematopoietic and endothelial markers such as CD45, CD34, CD11b or CD14, CD79 $\alpha$ or CD19 and HLA-DR surface molecules (11). Unlike embryonic stem cells and induced pluripotent stem cells, MSCs have a wide range of sources without concerns of ethic and teratoma formation, so they are considered promising materials for cell therapy in clinic $(8,12,13)$.

The first clinical trial of MSCs occurred in 1995. MSCs were applied as cellular pharmaceutical preparation for 15 patients with hematologic malignancies by autotransplantation after adherent culture in vitro, and no adverse reactions were observed in application (14). Since then, the research and clinical applications of MSCs have been booming for their properties of self-renewal and multi-lineage differentiation potential $(3,15-19)$. It is noteworthy that as a potential treatment, MSCs were used to prevent and reverse the cytokine storm caused by severe COVID-19 pneumonia for their comprehensive powerful functions in immunomodulatory and tissue injury repair $(20,21)$. As of August 2020, around the world, more than 1172 clinical trials using MSCs were registered on ClinicalTrials.gov. The related diseases involve myocardial infarction, graft versus host disease, diabetes, liver cirrhosis, spinal cord injury, osteoarthritis, Crobn's disease, multiple sclerosis, brain injury and COVID-19 pneumonia.

Generally, to acquire enough MSCs for therapy in clinic, MSCs are isolated from human tissues and 2D cultured in monolayer for fast expansion. However, numerous investigations demonstrated that the stemness of MSCs was gradually weakened with increased number of cell passage. The causative cell aging, reduced paracrine capacity as well as low survival rate after transplantation led to bad therapeutic effects $(22,23)$. Therefore, the 2D-cultured MSCs need to be optimized to improve cell quality before clinical application. In 2009, Lee et al. found that the intravenous hMSCs did not secrete the antiinflammatory protein TSG-6 until cells were embolized in the lung and this improved myocardial infarction in mice (24), suggesting that 3D MSCs aggregates benefit the implementation of MSC function. From that time on, 3D-cultured MSCs have been considered effective therapeutic agent in in vitro experiments and pre-clinical therapeutics by scientists and clinicians $(6,25-27)$. So far, it has been widely recognized that long-terms of 2D culture can change the original characteristics and traits of MSCs, while 3D culture can better preserve MSC properties due to creating a more natural physiological microenvironment (28-30).

Cell 3D culture methods can be classified as scaffoldbased and scaffold-free culture systems (31). For scaffoldbased 3D culture, cells are embedded in hydrogels or cultured in the presence of fibers or sponge-like structures to provide support, nutrition and extracellular matrix (ECM). Scaffold-free 3D culture allows the cells to selfassemble into spheroids to mimic the microenvironments in vivo by their own ECM. Compared with scaffold-based culture, scaffold-free 3D culture is more widely used in cell therapy research for its unique merits. 3D spheroid culture can endow MSCs the ability to tolerate the stresses in vivo, such as inflammatory microenvironment, hypoxia, nutrient depletion, which improves the survival of MSCs in vivo and enhances their beneficial function on damaged tissues. Moreover, the spheroids are generally formed with consistent size and shape without the need of dissolving the special scaffold that may cause immune response problems, which benefits high-throughput screening and optimization of MSCs as functional units for the demand of tissue engineering. Optimization of cell-based therapy by spheroid culture of MSCs has been widely recognized because it can maintain stemness, improve the ability of anti-apoptosis and homing post-transplantation, enhance anti-inflammatory and immunomodulatory effects, and increase paracrine capacity compared to traditional 2D-cultured MSCs $(13,32,33)$.

In this review, we will discuss the biological features and the medical applications of scaffold-free MSC spheroids, summarize different culture methods for MSC spheroid formation, and evaluate their advantages and disadvantages for application. We will interpret the internal interactions and microenvironment of MSCs that may contribute to aggregations, and illustrate the biological optimization of MSCs for their functions in spheroid cultures and its therapeutic effect in pre-clinical animal models. In the end, we will prospect the trends of this research field and the therapeutic challenges in the future.

\section{The mechanism of MSC spheroid formation in vitro}

In vitro, although the exact mechanism needs to be further elucidated, it has been accepted that the process of multicellular spheroid formation is through three steps, including loose cell aggregation via integrin-ECM binding, cadherin accumulation, and spheroid compaction (30). After self-assembly of MSCs via integrin-ECM binding or homophilic cadherin-cadherin interactions, cells will be reorganized to form aggregates for minimizing the free energy (32). Calcium-dependent interactions between cells and E-cadherin are key inducers to mediate MSC 3D-spheroid formation (6). From the morphologic view, high-cadherin-expression MSCs interact inside with round shape, and high-integrin-expression MSCs fully stretch outside with a spindle shape by intracellular tension of the aggregates, indicating the existence of mechanical polarization $(32,34,35)$. Endogenous ECM enhances the formation of MSC aggregates (36), and actin mainly mediates the viscoelastic behavior to promote fusion and compaction (35). In addition, the volume of cells in MSC spheroids is obviously reduced by $75-90 \%$ compared to that of traditional 2D-cultured MSCs $(26,37)$.

\section{The methods for MSC spheroid culture}

Spheroid culture artificially creates an environment permitting cells to interact with their surroundings in complete three dimensions, which may better preserve 
(a)

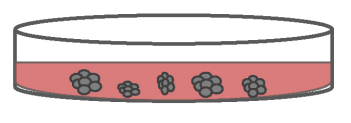

(b)

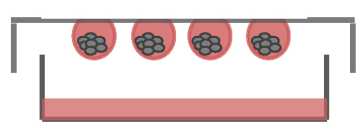

(c)

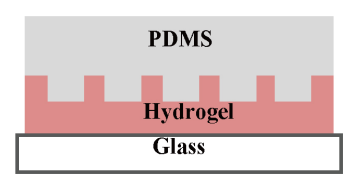

(d)

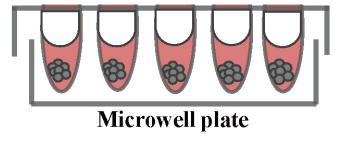

(e)
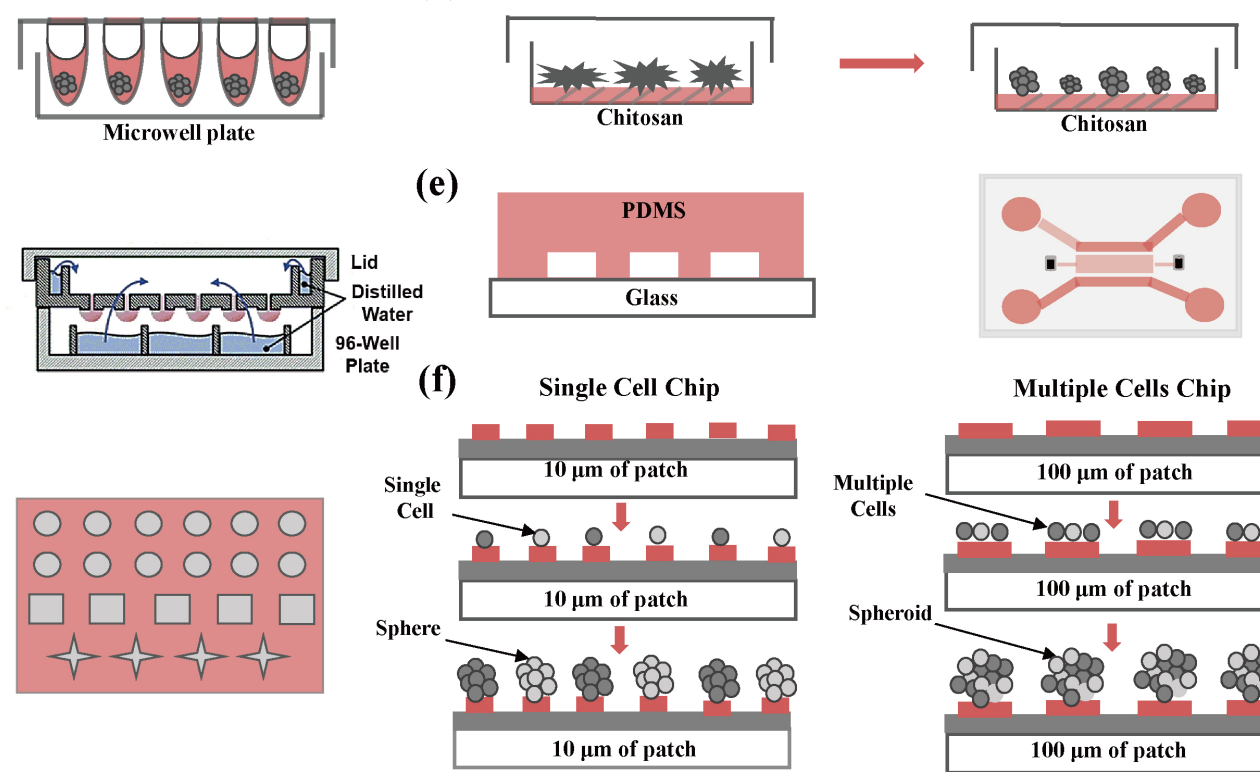
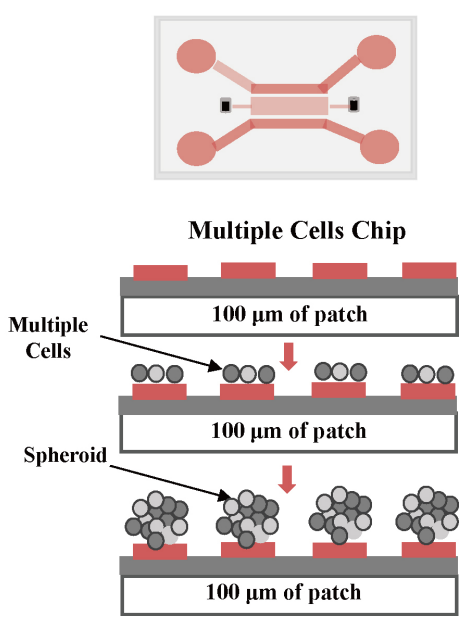

Figure 1. Methods used to generate MSC spheroids. (a) Overlay/Non-adhesive surface culture and ultra-low attachment microwell plates. (b) Gravity-induced spheroids by hanging drop culture and modified strategy (46). (c) Micropatterned substrates by micro-molding techniques using polydimethylsiloxane (PDMS). (d) Chitosan-mediated spheroid formation after transient adherence. (e) Microfluidic system (47). (f) Single cell derived sphere formation (45).

MSC properties due to mimicking a natural physiological microenvironment. Therefore, it is widely regarded as a preferable way to optimize MSCs (38-40). So far, many different methods have been developed to form MSC spheroids (Figure 1), such as non-adhesive surface (6), hanging drop (41), spinner flask (42), magnetic levitation (43) and chitosan-mediated (44). Especially, a novel method regarding single cell derived sphere (SCDS) formation on cell chip based on self-renewal screening dogma was developed recently, which exhibited its unique merits and shed new light in this research field (45). Although the characterization and properties of spheroids produced can be changed with different fabrications as well as different tissue sources, they all demonstrate their advantages to correspond to different treatment models.

Overlay/non-adhesive surface culture is commonly used for cell spheroid formation (Figure 1a). Suspended cells are seeded on plates coated with non-adhesive substrates, e.g. agarose gel/film (48), polyacrylamide gels (49), polyHEMA (50) or in ultra-low attachment culture dishes $(6,51)$. It is easy to operate but forming non-uniform spheroid size and shape. Lee et al. reported that different sized human umbilical cord blood-derived MSC (hUCB-MSC) spheroids were fabricated through suspension culture and obtained the spheroids by filtration using $100 \mu \mathrm{m}$ and 40 $\mu \mathrm{m}$ mesh strainer. After transplantation to rat myocardial infarction (MI) model, 3D-bullet of hUCB-MSCs had better therapeutic effect than 2D-cultured cells (6). Now, commercial microwell plate with ultra-low attachment has been developed to control the numbers and size of cell aggregates $(35,52)$. However, all these methods still cannot be used for mass production.

Hanging drop cell culture method is the most widely used technique for spheroid formation (Figure 1b). Cells were plated in hanging drops in $35-40 \mu \mathrm{L}$ of suspension containing 10,000-25,000 cells/drop for 3-4 days of culture to spontaneously form cell aggregates $(26,53)$. MSC spheroids generated by this method was used to enhance cartilage repair in a monosodium iodoacetate-induced osteoarthritis rat model (7) and to alleviate the hepatic injury resulted from ischemia-reperfusion (54). Another improved 384 hanging drop array for high-throughput 3D spheroid culture was developed for drug testing by robot. In this method, drops with cells are added to the hydrophilic plate surface that can be quickly attracted to form a hanging drop and confined within the plateau (46). These kinds of methods can produce uniform sized spheroids due to the controllable number of cells for spheroid formation, whereas it is difficult to obtain high yield of spheroids.

Micropatterned substrates are technologies for spheroid generation usually based on photolithography (Figure 1c). Polydimethylsiloxane (PDMS) stamps are used to mold a layer of polymer (e.g., HA/PEG/polystyrene) and fabricate controlled microstructures $(25,55,56)$. The micropatterned plates were used to generate MSC spheroids exogenously expressed with brain-derived neurotrophic factor (BDNF). These spheroids significantly improved recovery of hindlimb motor function in spinal cord injury (SCI) (57). Micropatterns can be manufactured in various sizes and shapes to meet different experimental requirements. Aggregates can be achieved and evaluated with high throughput but it also needs specialized facilities.

Chitosan-mediated spheroid formation is a new popular substrate-mediated method to generate 3D spheroids (Figure 1d). As a natural alkaline polysaccharide, chitosan is considered as a biocompatible and chemically 
modified polymer containing free amino groups. For this method, acetic acid solution contain $1 \%$ chitosan was coated on coverslip glass to form chitosan membranes. Hyaluronan, a kind of acid mucopolysaccharide, is added on chitosan membranes for increasing biological characteristics of spheroids. With positive charge, chitosan can easily form a complex with the strong negatively charged hyaluronan (58). Human adipose tissue derived MSC spheroids generated from the chitosan-based substrates could maintain the stemness and enhance the ability of cartilage differentiation (58), and showed higher cellular retention ratio than dissociated $2 \mathrm{D}$ cells after injected into muscle of nude mice (44). Formation of MSC spheroids on chitosan substrates is a little different from other common non-adhesive bio-membranes. Cells adhere transitorily on the chitosan membranes by their degree of deacetylation and then gradually aggregate to form spheroids. However, the sizes of spheroids cannot be even and this method cannot produce spheroids on a large scale.

Microfluidic system with microfluidic chips as the reaction devices can produce $3 \mathrm{D}$ cell spheroids by microwell (59) or microchannel (60) (Figure 1e). It was reported that the MSC spheroids could rapidly be formed in the controllable microenvironment with enhanced ability of osteogenic differentiation after encapsulated into hydrogel (60). In a 3D microfluidic system, glial cell-derived neurotrophic factor overexpressing hMSCs and human neural stem cells were co-cultured, leading to reduced glial differentiation and obviously enhanced neuronal differentiation (47). For this type of method, the most commonly used processing material is PDMS for its good biocompatibility and breathability. Although 3D culture on the chip can simulate the dynamic microenvironment in vivo with reduced reagent consumption, with small volumes, it is difficult to collect spheroids for subsequent analysis and treatment. Moreover, it needs complex chip design and operational control (61).

SCDS formation via cell array chip was recently developed using umbilical cord mesenchymal stem cells (UCMSCs) by Suo research team (45). This novel method is totally dependent on single cell $3 \mathrm{D}$ proliferation to form spheres, which is different from the above-mentioned 3D culture methods for multiple cell derived spheroid (MCDS) formations that are dependent on cells aggregation (Figure 1f). For SCDS formation, the cell chips are stamped with $10 \mu \mathrm{m}$ of diameter of polyethylenimine patches and $50 \mu \mathrm{m}$ of gap between two neighboring patches. One single cell is just allowed to attach on one patch. Therefore, single MSCs can be patterned on a chip by $2 \mathrm{D}$ and cultured to form SCDS by 3D. Since SCDS formation is based on the screening or training of cell self-renewal ability defined by single cell proliferation, it possesses some unique advantages in many aspects, such as stemness maintenance, cell survival, stress resistance, angiogenesis and homing, which greatly meets clinical requirements. In previous research, SCDS cultured MSCs displayed great therapeutic potential on acute liver failure in mice (45). Otherwise, this method can produce SCDS with small and uniformed size which is conductive to direct intravenous injection administration without further cell dissociation and avoids the heterogeneity problem of MSCs to a greater extent, therefore has potential for standardized production in large scale in the future. Remarkably, SCDS culture is completely superior to 2D culture and MCDS culture in all the above properties for MSC optimization (45). The study suggested that the microenvironment for SCDS formation can stimulate the self-renewal potential of MSCs and then optimizes MSCs. Although MCDS is generated by the aggregation of multiple cells, it may also provide a microenvironment in spheroids to promote SCDS formation and expansion, and then to enhance MSC stemness properties as well. However, the relatively enclosed environment generated by interactions among SCDSs may weaken the viability and stemness of MSCs in the entire MCDS, which suggests that the smaller size of MCDS may lead to better effect of MSC optimization. Despite many advantages of SCDS, there are still some mechanisms need to be further explored, for example, how SCDS culture impacts on MSC inflammatory response, paracrine capacities, immunomodulatory capacities, and cellular metabolism. In addition, more efforts need to be made to solve the problems regarding massive production.

\section{Spheroid culture creates a natural physiological microenvironment}

In multicellular organisms, every cell is surrounded by other cells and ECM to form a complex cellular society through cell communication, adhesion, junction and cell interaction. Cell social connections influence the behavior and fate of cells, as well as morphological structure and function. Moreover, physical effects of the in vivo microenvironment are important for stem cells and matrix elasticity to direct MSC lineage specification (49). In traditional 2D cell culture, cells are stretched and grown on flat plastic petri dishes, whereas in spheroid culture system, cells are aggregated into spheroids spontaneously or by force. So, for 2D cells, little areas are exposed to other cells and nearly half surface areas are exposed to stiff plastic culture dish (range of stiffness is 1-10 GPa), the other half are exposed to fluid medium $(62,63)$. However, for 3D spheroid cultured cells, almost all surface areas are covered via cell-cell contact and cell-ECM contact, which is closer to physiological microenvironment. Moreover, complex proteins such as collagen and elastin participate in the formation of ECM structure, contributing to the elasticity of spheroids. It was reported that typical elastic modulus of single cells in spheroids is approximately 103 $\mathrm{Pa}$ (64), which is far softer than 2D culture condition. Cell communication and signal transduction in spheroids are also closer to in vivo condition than in 2D culture.

\section{Microenvironment inside spheroid affects the cell viability}

3D-cultured cell spheroids have the potential to simulate the microenvironment and physiological activities in vivo. Cell spheroid has been used as a physiological model to 
study the occurrence of solid tumors and the differentiation of stem cells (30). Many mathematical and experimental models have been developed to simulate the transport of oxygen, carbon dioxide, nutrients and metabolic waste inside cell spheroids (65-67). When the diameter of cell spheroid is greater than $500 \mu \mathrm{m}$, there will be a threelayer structure with different proliferation states, including the marginal zone with proliferative and metabolic activity, the intermediate layer keeping quiescence and the necrotic core zone (30). Due to limited diffusion capacity, metabolic waste accumulated in the central layer of the multicellular spheroid may cause cell necrosis. Although the diameter of $100-150 \mu \mathrm{m}$ is considered the stable size that is adopted for simulating tumor spheroids based on the consideration of oxygen and nutrition limitation (68), it may not be appropriate for MSCs since MSCs typically reside in niches with much lower physiological oxygen tensions than cancer cells $(66,69)$. One recent study showed that $353 \pm 18 \mu \mathrm{m}$ diameter of MSC aggregates composed of up to $60,000 \mathrm{MSC}$ did not exhibit an obvious hypoxic core. The oxygen tension values from the outside edge of spheroids to the center varied less than $10 \%$, but cellular metabolism was decreased with increasing cell numbers and spheroid size. This may result from the decreased packing density and ECM deposition with increasing spheroid size. Moreover, caspase 3/7 activity was increased with spheroid size especially over 500 $\mu \mathrm{m}$ in diameter, but Annexin $\mathrm{V}$ was not detected in these spheroids (66). These results indicated that the enhanced function of MSC spheroids is not oxygen mediated, which contradicts many hypotheses that spheroid formation potentiates cell function by generating a hypoxic core within spheroids with large diameters. In addition, it is also consistent with the SCDS study that suggests that MCDS formed by aggregation of fewer cells and expansion to smaller size (no more than $50 \mu \mathrm{m}$ ) may maintain more stemness owing to the better microenvironment for cell metabolism and self-renewal (45).

\section{The mechanism by which spheroid culture better preserves MSC phenotype and innate properties}

Spheroids can maintain the stemness of MSCs and endow them strong multipotency. Spheroid-cultured MSCs exhibited higher colony-forming efficiency than 2D-cultured MSCs $(26,44,70)$. The expression of stem cell markers and genes related to self-renewal, such as Nanog, Sox2, Oct4, Klf4, SSEA-4 and $\beta$-catenin, are significantly increased in MSC spheroid cultures (44, 45, 70-72). Ling et al. reported that $3 \mathrm{D}$ spheroid culture of MSCs showed elevated expression levels of histone H3K9 acetylation in the promoter regions of Oct4, Nanog and Sox2. Moreover, the expression of microRNAs involved in stem cell potency was changed in accordance with the expression of these stemness genes (70). Release of actin cytoskeleton tension promotes Nanog expression, together with Suv39h1 (H3K9 methyltransferase) and H3K9me3 expression down-regulation in 3D spheroid formation (53). Compared with monolayer culture, the spheroid culture significantly upregulated the expression of Runx 2 and osteocalcin genes in MSCs after osteogenic induction (44, 72,73 ). It was reported that the differentiation capabilities of MSCs were significantly enhanced after spheroid formation, including increased trans-differentiation efficiency into neurons and hepatocyte-like cells by detecting the increased expression of Nestin and Albumin (44).

Spheroid culture is effective in improving the paracrine activity of MSCs (4). Spheroid-cultured MSCs secreted larger amounts of angiogenesis-inducing and immunosuppressive factors than 2D-cultured MSCs, such as VEGF, bFGF, angiogenin, IL-6, IL-8, IL-18, IL24, PGE2, CCL2, CCL5, TSG-6 and STC-1 (4, 26, 41, 73, 74), which contributes to MSC application in cell therapy.

In addition, spheroid culture promotes the survival and migration ability of MSCs, which is important for MSCs to home the lesion and function effectively in vivo $(35,75)$. Formation of MSC spheroids restores the functional expression of CXCR4 that is well known as an alphachemokine receptor specific for SDF- $1 \alpha$, a crucial mediator of cell migration $(35,76)$. The study found that more extracellular molecules were produced by spheroids than by 2D-cultured MSCs, such as laminin and fibronectin that contributes to cell migration and survival (44). When MSCs aggregate into spheroids, the expression of ECM proteins is increased and the E-cadherin mediated cell-cell adhesion is enhanced, which activates the $\mathrm{pEPK}$ and PI3K pathways, regulates higher levels of SOD2 to decrease oxidative stress and then facilitates cell survival $(7,33,42)$. Several studies demonstrated that MSC spheroids improved their potential by expressing some genes at higher levels, for instance, CXCR4 that increases the ability of homing to the inflammatory sites and adherence to endothelial cells, IL-24 that inhibits tumor growth and TSG-6 that has antiinflammatory activity $(26,76)$. Moreover, under hypoxic environment, spheroid culture enhanced expression of SDF$1 \alpha$ and HIF- $1 \alpha$, thereby enhancing MSC stress resistance, survival, homing and angiogenesis in vivo $(42,45)$.

Generally, adult stem cells are normally quiescent in the tissue, but can be activated for growth, migration and differentiation under changed microenvironment such as injury $(77,78)$. The niche in vivo helps to keep MSCs in quiescence, which benefits the maintenance of stem properties such as self-renewal (79). Due to better mimicking the properties of MSC niche, spheroid culture can promote the MSCs to enter G0 phase, which contributes to the recovery of the original characteristics of MSCs such as mesenchymal trait, stemness, selfrenewal and migration ability that have been lost during long-term of 2D culture (45). Quiescence also endows MSCs the ability to adapt to hostile environment such as hypoxia, nutrition starvation and ECM-detachment, eventually enhancing MSC survival. The AMP-activated protein kinase (AMPK)/ mammalian target of rapamycin (mTORC) signaling pathway may be involved in spheroid culture of MSCs (45). AMPK is a crucial metabolic regulator to maintain cellular energy homeostasis and thereby benefits cell survival under stresses. The role of mTORC1 is to activate translation of proteins and then contributes to cell proliferation (80). Spheroid culture 
significantly increased the activity of AMPK and decreased the mTORC activity. This may explain why spheroid culture can enhance the quiescence of MSCs. Recently, evidence has increasingly shown that mTORC1 is a crucial factor to induce and maintain the cell senescent state $(81,82)$. The decreased mTORC1 activity may delay the senescence occurrence in MSCs.

\section{Application of MSC spheroids in pre-clinical studies}

Spheroids have greater advantages in survival, factor secretion, maintenance of stemness, migration, anti-aging, anti-inflammatory and angiogenesis than normal adherentcultured cells, indicating their good prospects of clinical application in tissue wound-healing and regeneration for inflammatory diseases and common diseases including bone and cartilage injury $(72,83)$, tissue inflammation $(26$, $45,57,74)$, cardiovascular infarction (6) and ischemic injury $(33,42,54)$. For bone and cartilage injury, one study reported that the transplantation of 3D spheroids of mouse bone marrow derived MSCs rapidly promoted new bone regeneration in aged mice with a calvarial defect through activation of the Wnt/ $\beta$-catenin and Smad signal pathways (72). In this study, 3D spheroid MSCs expressed higher levels of stemness markers and upregulated expression of osteogenesis-related molecules than 2D monolayer MSCs in vitro. It was also reported that the implantation of synovium MSC spheroids at relatively low density improved chondrogenesis and subsequent cartilage formation. Aggregates of MSCs up-regulated the expression of chondrogenic genes (SOX9, COL2A1 and $A C A N)$ and exhibited better cartilage repair than $2 \mathrm{D}$ culture $(83,84)$. For cardiovascular infarction and ischemic injury, it was reported that the transplantation of UCMSC spheroids was superior to cells from conventional 2D culture in therapeutic efficacy for rat $\mathrm{MI}$ model. As a result, the fibrosis area was smaller, infarct wall was thicker, capillary density was significantly higher and contractility was improved (6). Another study reported that spheroid cultured human adipose-derived MSCs significantly reduced the rate of limb loss in ischemic tissue owing to the enhanced angiogenesis by higher level of angiogenic growth factor secretion than 2D-cultured cells (42). This is consistent with other studies in which the spheroid culture of MSCs showed better ability of angiogenesis than 2D-cultured MSCs $(33,45)$. For other injuries, it was found that UCMSC spheroids were better than 2D-cultured UCMSCs for therapy of acute liver failure in mouse model due to the reduced expression of tumor necrosis factor- $\alpha$ (TNF- $\alpha)$ and alpha smooth muscle actin $(\alpha-$ SMA $)(45,71)$. In a study regarding SCI in mice, BDNF-transfected MSC spheroids significantly enhanced the motor function recovery with reduced damage to spinal neurons, while conventional 2D-cultured MSCs failed to exert therapeutic effects (57). In summary, it is of more importance to apply spheroid cultured MSCs for regenerative medical studies at present.

\section{Limitation and difficulties of MSC spheroid}

\section{application in clinic}

Although most of the studies support that MSCs can be optimized by 3D spheroid culture, under some situations, spheroids may be not better than 2D-cultured cells for application (85-89). These studies indicate that MSC spheroids need to be used in suitable conditions, and indepth understanding about key molecules responsible for the cell fate control of MSCs during spheroid formation is needed. In addition, spheroid culture promotes the MSCs to enter G0 phase, which inhibits the expansion of cells although quiescence contributes to the maintenance of cell stemness. Therefore, more reasonable methods need to be developed to optimize the MSCs. Recently, Yan et al. reported a new method for efficient large-scale hMSC expansion with low indications of senescence phenotype and stable characteristics for high cell recovery rate (90). However, this method cannot realize the optimization of MSCs to enhance MSC properties as spheroid formation did.

Although the existing methods of spheroid formation can optimize MSCs, they generally cannot avoid cell dissociation, uneven quality and non-uniform size, which prevents high-quality optimization and standardized production of MSCs, and impedes the application of MSC spheroids in clinic. The method of MSC SCDS formation based on self-renew screening has tried to overcome the above shortcomings, but more efforts need to be made to realize the cell expansion. Furthermore, once the clinical applications are involved, standardized, largescale and uniform MSC spheroid culture is required, and the corresponding detection methods for spheroid identification, quality inspection and evaluation are also essential. However, these problems have not been fully resolved so far.

\section{Conclusions and perspectives}

Spheroid cultured MSCs have shown the abilities of anti-apoptosis, enhanced stemness, increased sections, anti-inflammatory and immunomodulatory properties as well as induced angiogenesis in vitro and in vivo. These features indicate their unique therapeutic potential in tissue damage, regenerative medicine and inflammatory diseases, however, a wealth of problems need to be solved in the future. First, the in-depth molecular mechanisms by which crucial regulators, signaling pathways and biological processes are involved in MSC survival, senescence, stemness, homing, factor secretion and differentiation should be uncovered, as well as the relations between spheroid structure and cell function. Actually, gene expression differences between MSC spheroids and 2D cultures have been widely detected in various spheroid investigations, but little has been done for further deep explorations. Second, optimized MSC spheroids for clinical applications should meet the demands of uniform size, reproducibility, low cell heterogeneity, stable properties, easy operation for preparation, and large-scale and standardized production. Third, the optimal dosing strategy and administration route of 3D MSC spheroids need to be investigated in preclinical research. Fourth, more clinical trials need to be 
performed to verify the good therapeutic potential of MSC spheroids. Given the big size of spheroids preventing them from vein administration, the therapeutic advantages may be first fully exerted in skin tissue repair of clinical wounds, ulcers, burns, scars, surgical defects and deformities, in which the MSC spheroids can be applied without relying on vein administration.

\section{Acknowledgement}

Livingchip Inc. is acknowledged for technical consulting of cell spheroid culture and screening. This research was supported by funds from the Strategic Priority Research Program of the Chinese Academy of Sciences (Grant XDA16020103), Natura Science Foundation of Jiangxi Province (20192ACBL20053), the Ministry of Science and Technology (MOST) of China (Grant 2017YFA0104301, 2018YFA0107900), National Natura Science Foundation of China (Grant 31870975, 31771491), Suzhou Science and Technology Development Program (Grant SYG201835), the National Nature Science Foundation and Shanghai Municipal Government (Grant, 2019CXJQ01).

\section{Conflict of interest}

The authors declare that there is no conflict of interests regarding the publication of this paper.

\section{References}

1. Friedenstein AJ, Chailakhjan RK, Lalykina KS. The development of fibroblast colonies in monolayer cultures of guinea-pig bone marrow and spleen cells. Cell Tissue Kinet. 1970;3(4):393-403.

2. Wei X, Yang X, Han ZP, Qu FF, Shao L, Shi YF. Mesenchymal stem cells: a new trend for cell therapy. Acta Pharmacol Sin. 2013;34(6):747-54.

3. Sharma RR, Pollock K, Hubel A, McKenna D. Mesenchymal stem or stromal cells: a review of clinical applications and manufacturing practices. Transfusion. 2014;54(5):1418-37.

4. Miceli V, Pampalone M, Vella S, Carreca AP, Amico G, Conaldi PG. Comparison of immunosuppressive and angiogenic properties of human amnion-derived mesenchymal stem cells between $2 \mathrm{D}$ and $3 \mathrm{D}$ culture systems. Stem Cells Int. 2019;2019:7486279.

5. Pisciotta A, Bertoni L, Riccio M, Mapelli J, Bigiani A, La Noce $M$, et al. Use of a 3D floating sphere culture system to maintain the neural crest-related properties of human dental pulp stem cells. Front Physiol. 2018;9:547-47.

6. Lee EJ, Park SJ, Kang SK, Kim GH, Kang HJ, Lee SW, et al. Spherical bullet formation via E-cadherin promotes therapeutic potency of mesenchymal stem cells derived from human umbilical cord blood for myocardial infarction. Mol Ther. 2012;20(7):1424-33.

7. Lee M, Song BR, Kim DH, Ha J, Lee M, Choi SJ, et al. Up-regulation of superoxide dismutase 2 in 3D spheroid formation promotes therapeutic potency of human umbilical cord blood-derived mesenchymal stem cells. Antioxidants. 2020;9(1)

8. Aggarwal S, Pittenger MF. Human mesenchymal stem cells modulate allogeneic immune cell responses. Blood. 2005;105(4):1815-22

9. Caplan Al, Dennis JE. Mesenchymal stem cells as trophic mediators. J Cell Biochem. 2006;98(5):1076-84

10. Uccelli A, Moretta L, Pistoia V. Mesenchymal stem cells in health and disease. Nat Rev Immunol. 2008;8(9):726-36.

11. Dominici M, Le Blanc K, Mueller I, Slaper-Cortenbach I, Marini F, Krause D, et al. Minimal criteria for defining multipotent mesenchymal stromal cells. The International Society for Cellular Therapy position statement. Cytotherapy.
2006;8(4):315-7

12. Takahashi K, Yamanaka S. Induction of pluripotent stem cells from mouse embryonic and adult fibroblast cultures by defined factors. Cell. 2006;126(4):663-76.

13. Hare JM, Traverse JH, Henry TD, Dib N, Strumpf RK, Schulman SP, et al. A randomized, double-blind, placebocontrolled, dose-escalation study of intravenous adult human mesenchymal stem cells (prochymal) after acute myocardial infarction. J Am Coll Cardiol. 2009;54(24):2277-86.

14. Lazarus HM, Haynesworth SE, Gerson SL, Rosenthal NS, Caplan Al. Ex vivo expansion and subsequent infusion of human bone marrow-derived stromal progenitor cells (mesenchymal progenitor cells): implications for therapeutic use. Bone Marrow Transplant. 1995;16(4):557-64.

15. Horwitz EM, Prockop DJ, Fitzpatrick LA, Koo WW, Gordon $\mathrm{PL}$, Neel M, et al. Transplantability and therapeutic effects of bone marrow-derived mesenchymal cells in children with osteogenesis imperfecta. Nat Med. 1999;5(3):309-13.

16. Zuk PA, Zhu M, Mizuno H, Huang J, Futrell JW, Katz AJ, et al. Multilineage cells from human adipose tissue: implications for cell-based therapies. Tissue Eng. 2001;7(2):211-28.

17. Horwitz EM, Gordon PL, Koo WK, Marx JC, Neel MD, McNall RY, et al. Isolated allogeneic bone marrow-derived mesenchymal cells engraft and stimulate growth in children with osteogenesis imperfecta: Implications for cell therapy of bone. Proc Natl Acad Sci U S A. 2002;99(13):8932-7.

18. Togel F, Hu Z, Weiss K, Isaac J, Lange C, Westenfelder C. Administered mesenchymal stem cells protect against ischemic acute renal failure through differentiationindependent mechanisms. Am J Physiol Renal Physiol. 2005;289(1):F31-42.

19. LeBlanc K, Frassoni F, Ball L, Locatelli F, Roelofs H, Lewis I, et al. Mesenchymal stem cells for treatment of steroidresistant, severe, acute graft-versus-host disease: a phase II study. Lancet. 2008;371(9624):1579-86.

20. Golchin A, Seyedjafari E, Ardeshirylajimi A. Mesenchymal stem cell therapy for COVID-19: present or future. Stem Cell Rev Rep. 2020;16(3):427-33.

21. Leng Z, Zhu R, Hou W, Feng Y, Yang Y, Han Q, et al. Transplantation of ACE2(-) Mesenchymal stem cells improves the outcome of patients with COVID-19 pneumonia. Aging Dis. 2020;11(2):216-28.

22. Galipeau J. The mesenchymal stromal cells dilemma--does a negative phase III trial of random donor mesenchymal stromal cells in steroid-resistant graft-versus-host disease represent a death knell or a bump in the road? Cytotherapy. 2013;15(1):2-8.

23. Regmi S, Pathak S, Kim JO, Yong CS, Jeong JH Mesenchymal stem cell therapy for the treatment of inflammatory diseases: challenges, opportunities, and future perspectives. Eur J Cell Biol. 2019;98(5-8):151041.

24. Lee RH, Pulin AA, Seo MJ, Kota DJ, Ylostalo J, Larson BL, et al. Intravenous hMSCs improve myocardial infarction in mice because cells embolized in lung are activated to secrete the anti-inflammatory protein TSG-6. Cell Stem Cell. 2009;5(1):54-63.

25. Wang W, Itaka K, Ohba S, Nishiyama N, Chung UI, Yamasaki $Y$, et al. 3D spheroid culture system on micropatterned substrates for improved differentiation efficiency of multipotent mesenchymal stem cells. Biomaterials. 2009;30(14):2705-15.

26. Bartosh TJ, Ylostalo JH, Mohammadipoor A, Bazhanov $\mathrm{N}$, Coble K, Claypool K, et al. Aggregation of human mesenchymal stromal cells (MSCs) into 3D spheroids enhances their antiinflammatory properties. Proc Natl Acad Sci U S A. 2010;107(31):13724-9.

27. Frith JE, Thomson B, Genever PG. Dynamic threedimensional culture methods enhance mesenchymal stem cell properties and increase therapeutic potential. Tissue Eng Part C Methods. 2010;16(4):735-49.

28. Morikawa S, Mabuchi Y, Kubota Y, Nagai Y, Niibe K, Hiratsu 
E, et al. Prospective identification, isolation, and systemic transplantation of multipotent mesenchymal stem cells in murine bone marrow. J Exp Med. 2009;206(11):2483-96.

29. Fennema E, Rivron N, Rouwkema J, van Blitterswijk C, de Boer J. Spheroid culture as a tool for creating 3D complex tissues. Trends Biotechnol. 2013;31(2):108-15.

30. Lin RZ, Chang HY. Recent advances in three-dimensional multicellular spheroid culture for biomedical research. Biotechnol J. 2008;3(9-10):1172-84

31. Ovsianikov A, Khademhosseini A, Mironov V. The Synergy of Scaffold-Based and Scaffold-Free Tissue Engineering Strategies. Trends Biotechnol. 2018;36(4):348-57.

32. Sart S, Tsai AC, Li Y, Ma T. Three-dimensional aggregates of mesenchymal stem cells: cellular mechanisms, biologica properties, and applications. Tissue Eng Part B Rev. 2014;20(5):365-80.

33. Xu Y, Shi T, Xu A, Zhang L. 3D spheroid culture enhances survival and therapeutic capacities of MSCs injected into ischemic kidney. J Cell Mol Med. 2016;20(7):1203-13.

34. Peng R, Yao X, Cao B, Tang J, Ding J. The effect of culture conditions on the adipogenic and osteogenic inductions of mesenchymal stem cells on micropatterned surfaces. Biomaterials. 2012;33(26):6008-19.

35. Tsai AC, Liu Y, Yuan X, Ma T. Compaction, fusion, and functional activation of three-dimensional human mesenchymal stem cell aggregate. Tissue Eng Part A. 2015;21(9-10):1705-19.

36. Kim J, Ma T. Endogenous extracellular matrices enhance human mesenchymal stem cell aggregate formation and survival. Biotechnol Prog. 2013;29(2):441-51.

37. Ezquer F, Morales P, Quintanilla ME, Santapau D, LespayRebolledo C, Ezquer M, et al. Intravenous administration of anti-inflammatory mesenchymal stem cell spheroids reduces chronic alcohol intake and abolishes binge-drinking. Sci Rep. 2018;8(1):4325.

38. Antoni D, Burckel H, Josset E, Noel G. Three-dimensiona cell culture: a breakthrough in vivo. Int $\mathrm{J}$ Mol Sci. 2015;16(3):5517-27.

39. Edmondson R, Broglie JJ, Adcock AF, Yang L. Threedimensional cell culture systems and their applications in drug discovery and cell-based biosensors. Assay Drug Dev Technol. 2014;12(4):207-18.

40. Lee J, Cuddihy MJ, Kotov NA. Three-dimensional cel culture matrices: state of the art. Tissue Eng Part B Rev. 2008;14(1):61-86.

41. Potapova IA, Gaudette GR, Brink PR, Robinson RB, Rosen MR, Cohen IS, et al. Mesenchymal stem cells support migration, extracellular matrix invasion, proliferation, and survival of endothelial cells in vitro. Stem Cells. 2007;25(7):1761-8.

42. Bhang SH, Cho SW, La WG, Lee TJ, Yang HS, Sun AY, et al. Angiogenesis in ischemic tissue produced by spheroid grafting of human adipose-derived stromal cells. Biomaterials. 2011;32(11):2734-47.

43. Lewis NS, Lewis EE, Mullin M, Wheadon H, Dalby MJ, Berry CC. Magnetically levitated mesenchymal stem cell spheroids cultured with a collagen gel maintain phenotype and quiescence. J Tissue Eng. 2017;8:2041731417704428.

44. Cheng NC, Wang S, Young TH. The influence of spheroid formation of human adipose-derived stem cells on chitosan films on stemness and differentiation capabilities. Biomaterials. 2012;33(6):1748-58.

45. Qiao Y, Xu Z, Yu Y, Hou S, Geng J, Xiao T, et al. Single cell derived spheres of umbilical cord mesenchymal stem cells enhance cell stemness properties, survival ability and therapeutic potential on liver failure. Biomaterials. 2020;227:119573.

46. Tung YC, Hsiao AY, Allen SG, Torisawa YS, Ho M, Takayama S. High-throughput 3D spheroid culture and drug testing using a 384 hanging drop array. Analyst. 2011;136(3):473-8.

47. Yang K, Park HJ, Han S, Lee J, Ko E, Kim J, et al.
Recapitulation of in vivo-like paracrine signals of human mesenchymal stem cells for functional neuronal differentiation of human neural stem cells in a 3D microfluidic system. Biomaterials. 2015;63:177-88.

48. Strainiene E, Binkis M, Urnikyte S, Stankevicius V, Sasnauskiene A, Kundrotas G, et al. Microenvironment dependent gene expression signatures in reprogrammed human colon normal and cancer cell lines. BMC cancer. 2018;18(1):222-22.

49. Engler AJ, Sen S, Sweeney HL, Discher DE. Matrix elasticity directs stem cell lineage specification. Cell. 2006;126(4):677-89.

50. Kim M, Yun HW, Park DY, Choi BH, Min BH. Threedimensional spheroid culture increases exosome secretion from mesenchymal stem cells. Tissue Eng Regen Med. 2018;15(4):427-36.

51. Tietze S, Krater M, Jacobi A, Taubenberger A, Herbig M, Wehner R, et al. Spheroid culture of mesenchymal stromal cells results in morphorheological properties appropriate for improved microcirculation. Adv Sci. 2019;6(8):1802104.

52. Baraniak PR, McDevitt TC. Scaffold-free culture of mesenchymal stem cell spheroids in suspension preserves multilineage potential. Cell Tissue Res. 2012;347(3):701-11.

53. Zhou Y, Chen H, Li H, Wu Y. 3D culture increases pluripotent gene expression in mesenchymal stem cells through relaxation of cytoskeleton tension. J Cell Mol Med. 2017;21(6):1073-84

54. Sun Y, Wang Y, Zhou L, Zou Y, Huang G, Gao G, et al. Spheroid-cultured human umbilical cord-derived mesenchymal stem cells attenuate hepatic ischemiareperfusion injury in rats. Sci Rep. 2018;8(1):2518.

55. Dusseiller MR, Schlaepfer D, Koch M, Kroschewsk $\mathrm{R}$, Textor M. An inverted microcontact printing method on topographically structured polystyrene chips for arrayed micro-3-D culturing of single cells. Biomaterials. 2005;26(29):5917-25.

56. Khademhosseini A, Eng G, Yeh J, Fukuda J, Blumling J, 3rd, Langer R, et al. Micromolding of photocrosslinkable hyaluronic acid for cell encapsulation and entrapment. J Biomed Mater Res A. 2006;79(3):522-32.

57. Uchida S, Hayakawa K, Ogata T, Tanaka S, Kataoka K, Itaka K. Treatment of spinal cord injury by an advanced cell transplantation technology using brain-derived neurotrophic factor-transfected mesenchymal stem cell spheroids. Biomaterials. 2016;109:1-11.

58. Huang GS, Dai LG, Yen BL, Hsu SH. Spheroid formation of mesenchymal stem cells on chitosan and chitosanhyaluronan membranes. Biomaterials. 2011;32(29):6929-45.

59. Wuchter P, Saffrich R, Giselbrecht S, Nies C, Lorig H, Kolb S, et al. Microcavity arrays as an in vitro model system of the bone marrow niche for hematopoietic stem cells. Cell and tissue research. 2016;364(3):573-84.

60. Chan HF, Zhang Y, Ho YP, Chiu YL, Jung Y, Leong KW Rapid formation of multicellular spheroids in doubleemulsion droplets with controllable microenvironment. Sci Rep. 2013;3:3462

61. Halldorsson S, Lucumi E, Gomez-Sjoberg R, Fleming RMT. Advantages and challenges of microfluidic cell culture in polydimethylsiloxane devices. Biosens Bioelectron. 2015;63:218-31

62. Page H, Flood P, Reynaud EG. Three-dimensional tissue cultures: current trends and beyond. Cell Tissue Res. 2013;352(1):123-31.

63. Akhmanova M, Osidak E, Domogatsky S, Rodin S Domogatskaya A. Physical, spatial, and molecular aspects of extracellular matrix of in vivo niches and artificial scaffolds relevant to stem cells research. Stem Cells Int. 2015;2015:167025.

64. Al-Agele R, Paul E, Taylor S, Watson C, Sturrock C, Drakopoulos $M$, et al. Physics of animal health: on the mechano-biology of hoof growth and form. J R Soc Interface. 2019;16(155):20190214. 
65. Derda R, Laromaine A, Mammoto A, Tang SK, Mammoto $\mathrm{T}$, Ingber DE, et al. Paper-supported 3D cell culture for tissue-based bioassays. Proc Natl Acad Sci U S A. 2009;106(44):18457-62.

66. Murphy KC, Hung BP, Browne-Bourne S, Zhou D, Yeung $J$, Genetos DC, et al. Measurement of oxygen tension within mesenchymal stem cell spheroids. J R Soc Interface. 2017;14(127).

67. Curcio E, Salerno S, Barbieri G, De Bartolo L, Drioli E, Bader A. Mass transfer and metabolic reactions in hepatocyte spheroids cultured in rotating wall gas-permeable membrane system. Biomaterials. 2007;28(36):5487-97.

68. Brophy CM, Luebke-Wheeler JL, Amiot BP, Khan H, Remme RP, Rinaldo $\mathrm{P}$, et al. Rat hepatocyte spheroids formed by rocked technique maintain differentiated hepatocyte gene expression and function. Hepatology. 2009;49(2):578-86.

69. Mohyeldin A, Garzon-Muvdi T, Quinones-Hinojosa A. Oxygen in stem cell biology: a critical component of the stem cell niche. Cell Stem Cell. 2010;7(2):150-61.

70. Guo L, Zhou Y, Wang S, Wu Y. Epigenetic changes of mesenchymal stem cells in three-dimensional (3D) spheroids. J Cell Mol Med. 2014;18(10):2009-19.

71. Li Y, Guo G, Li L, Chen F, Bao J, Shi YJ, et al. Threedimensional spheroid culture of human umbilical cord mesenchymal stem cells promotes cell yield and stemness maintenance. Cell Tissue Res. 2015;360(2):297-307.

72. Imamura A, Kajiya H, Fujisaki S, Maeshiba M, Yanagi $\mathrm{T}$, Kojima $\mathrm{H}$, et al. Three-dimensional spheroids of mesenchymal stem/stromal cells promote osteogenesis by activating stemness and Wnt/beta-catenin. Biochem Biophys Res Commun. 2020;523(2):458-64.

73. Park MJ, Lee J, Byeon JS, Jeong D-U, Gu N-Y, Cho I-S, et al. Effects of three-dimensional spheroid culture on equine mesenchymal stem cell plasticity. Vet Res Commun. 2018;42(3):171-81.

74. Murphy KC, Whitehead J, Falahee PC, Zhou D, Simon SI, Leach JK. Multifactorial experimental design to optimize the anti-inflammatory and proangiogenic potential of mesenchymal stem cell spheroids. Stem Cells. 2017;35(6):1493-504.

75. Huang GS, Hsieh PS, Tseng CS, Hsu SH. The substratedependent regeneration capacity of mesenchymal stem cell spheroids derived on various biomaterial surfaces. Biomater Sci. 2014;2(11):1652-60.

76. Potapova IA, Brink PR, Cohen IS, Doronin SV. Culturing of human mesenchymal stem cells as three-dimensional aggregates induces functional expression of CXCR4 that regulates adhesion to endothelial cells. J Biol Chem. 2008;283(19):13100-7.

77. Dhawan J, Laxman S. Decoding the stem cell quiescence cycle--lessons from yeast for regenerative biology. J Cell Sci. 2015;128(24):4467-74

78. Monge C, DiStasio N, Rossi T, Sebastien M, Sakai H, Kalman B, et al. Quiescence of human muscle stem cells is favored by culture on natural biopolymeric films. Stem Cell Res Ther. 2017;8(1):104.

79. Cheung TH, Rando TA. Molecular regulation of stem cell quiescence. Nat Rev Mol Cell Biol. 2013;14(6):329-40.

80. Ben-Sahra I, Manning BD. mTORC1 signaling and the metabolic control of cell growth. Curr Opin Cell Biol. 2017;45:72-82.

81. Nacarelli T, Sell C. Targeting metabolism in cellular senescence, a role for intervention. Mol Cell Endocrinol. 2017;455(C):83-92.

82. Carroll B, Nelson G, Rabanal-Ruiz Y, Kucheryavenko O, Dunhill-Turner NA, Chesterman CC, et al. Persistent mTORC1 signaling in cell senescence results from defects in amino acid and growth factor sensing. J Cell Biol. 2017;216(7):1949-57.

83. Suzuki S, Muneta T, Tsuji K, Ichinose S, Makino H, Umezawa $A$, et al. Properties and usefulness of aggregates of synovial mesenchymal stem cells as a source for cartilage regeneration. Arthritis Res Ther. 2012;14(3):R136.

84. Leijten J, Teixeira LSM, Bolander J, Ji W, Vanspauwen B, Lammertyn $\mathrm{J}$, et al. Bioinspired seeding of biomaterials using three dimensional microtissues induces chondrogenic stem cell differentiation and cartilage formation under growth factor free conditions. Scientific reports. 2016;6:36011-11.

85. Rajaraman G, White J, Tan KS, Ulrich D, Rosamilia A, Werkmeister $\mathrm{J}$, et al. Optimization and scale-up culture of human endometrial multipotent mesenchymal stromal cells: potential for clinical application. Tissue Eng Part C Methods. 2013;19(1):80-92.

86. Mauck RL, Yuan X, Tuan RS. Chondrogenic differentiation and functional maturation of bovine mesenchymal stem cells in long-term agarose culture. Osteoarthritis Cartilage. 2006;14(2):179-89.

87. Futrega K, Atkinson K, Lott WB, Doran MR. Spheroid coculture of hematopoietic stem/progenitor cells and monolayer expanded mesenchymal stem/stromal cells in polydimethylsiloxane microwells modestly improves in vitro hematopoietic stem/progenitor cell expansion. Tissue Eng Part C Methods. 2017;23(4):200-18.

88. Schmal O, Seifert J, Schaffer TE, Walter CB, Aicher WK, Klein G. Hematopoietic stem and progenitor cell expansion in contact with mesenchymal stromal cells in a hanging drop model uncovers disadvantages of 3D culture. Stem Cells Int. 2016;2016:4148093.

89. Huang W, Wang C, Xie L, Wang X, Zhang L, Chen C, et al. Traditional two-dimensional mesenchymal stem cells (MSCs) are better than spheroid MSCs on promoting retinal ganglion cells survival and axon regeneration. Exp Eye Res. 2019;185:107699.

90. Yan X, Zhang K, Yang Y, Deng D, Lyu C, Xu H, et al. Dispersible and dissolvable porous microcarrier tablets enable efficient large-scale human mesenchymal stem cell expansion. Tissue Eng Part C Methods. 2020;26(5):263-75. 